\title{
Efficient Use of Transmission Capacity for Cross- border Trading: Available Transfer Capacity versus Flow-based Approach
}

\author{
Glenn Plancke, Kristof De Vos, Cedric De Jonghe, Ronnie Belmans \\ KU Leuven, EnergyVille \\ Belgium
}

\begin{abstract}
The European Union strives towards an integrated European electricity market. Achieving this objective while preserving a zonal pricing approach in combination with market coupling, requires an efficient cross-border transmission capacity calculation and allocation mechanism. In this context, a new flowbased algorithm is developed in the CWE region for the dayahead time frame. The idea is to accurately take into account the impact each trade has on the grid by translating cross-border contractual exchanges into physical flows. This paper compares this flow-based approach to the conventional ATC mechanism for the four steps that can be distinguished in the daily cross-border coordination process. To this end, market reports concerning the operation of the flow-based method are studied. While flow-based market coupling is beneficial to ATC from a theoretical point of view, operational challenges before, during and after market coupling are identified. These have to be considered as well in assessing the overall improvement of flow-based compared to ATC.
\end{abstract}

Index Terms - Available Transfer Capacity (ATC), Electricity market, Flow-based (FB), Market Coupling, Transmission system integration

\section{INTRODUCTION}

The European electricity market is operated based on a zonal configuration ${ }^{1}$. Equal prices are derived for all nodes within a zone, neglecting internal network constraints. This zonal pricing mechanism is a simplification of nodal pricing where locational marginal prices (LMPs) are determined for each separate node of the network. Besides an energy component, these prices also reflect transmission constraints such as congestion and transmission losses. Nodal pricing is implemented in certain regions in the world, e.g. the PJM area in the US, Chile, New Zealand, and is often regarded as a benchmark for operational efficiency [1], [2]. For Europe however, a move towards nodal pricing is not foreseen in the near future, as the Target Model put forward by ENTSO-e still builds on the zonal approach.

Accurately representing the transmission network with a zonal pricing approach is challenging [3]. Besides determining the size of the zones, also the transmission capacity available for trade between zones has to be defined. Furthermore, the way in which this capacity is allocated has a substantial impact on the market [4]. The goal of this paper is to provide a better understanding of the flow-based mechanism by comparing the main features of this novel approach for capacity calculation and allocation to the commonly used Available Transfer Capacity (ATC) mechanism ${ }^{2}$. The analysis focusses on the dayahead time frame.

Cross-border electricity trading fundamentally requires a coordinated capacity calculation and allocation mechanism. Coordination across bidding zones is essential since power flows cannot be restricted by commercial agreements but follow the laws of physics [5]. For example, when Germany exports electricity to France, part of the electric power will flow through the Netherlands and Belgium, instead of following the direct path between the two countries. As such, this transaction also has an impact on the remaining interconnection capacity at the Dutch and Belgian borders.

The structure of the paper is as follows. Section 2 compares the main features of the capacity calculation and allocation process for the flow-based approach against the ATC methodology. Subsequently, section 3 elaborates on the main operational challenges of the flow-based mechanism before, during and after market coupling. Section 4 concludes.

\section{FLOW-BASED VERSUS AVAILABLE TRANSFER CAPACITY}

The goal of a coordinated capacity calculation and allocation mechanism in the context of cross-border electricity trading is to guarantee an efficient allocation of the available transmission capacity. A trade-off has to be made between offering capacity to the market and ensuring a reliable operation of the power system. The flow-based and ATC methodology are compared side by side in this section for the four steps that can be distinguished in the daily cross-border coordination process [6]-[8].

\section{A. Base Case: Calculation of Available Capacity}

Two days before delivery, each Transmission System Operator (TSO) creates a congestion forecast file (D2CF file) describing its part of the grid in the best way. The D2CF file provides a best estimate of the state of the system for day $\mathrm{D}$, containing minimally the following information:

- $\quad$ available grid topology, and anticipated outages of grid components;
1 The current bidding zone configuration in Europe mainly corresponds with national borders, except for Scandinavia and Italy which contain multiple price zones. On the other hand, Germany, Luxemburg and Austria constitute a single bidding zone.
${ }^{2}$ Flow-based market coupling went live for the day-ahead market in the CWE region on May $20^{\text {th }} 2015$ 
- $\quad$ generation units and their estimated output levels, and anticipated outages;

- forecast of load and renewable generation;

- best estimate of exchange programs.

Subsequently, the individual D2CF files from all TSOs are merged by Coreso, leading to the so called base case ${ }^{3}$. Coreso is involved in operational coordination services in cooperation with TSOs, while TSOs themselves remain responsible for operation.

\section{1) Available Transfer Capacity (ATC)}

Under ATC, based on the information contained in the base case, each Transmission System Operator (TSO) determines a Net Transfer Capacity (NTC) value for each direction on each border of its control area (Fig. 1). The NTC value can be interpreted as the maximum allowable commercial exchange between two zones that push at least one critical network element to its physical limit. At this stage, TSOs of neighbouring zones coordinate bilaterally to align the NTC values on their common border, generally selecting the lowest NTC.

$$
E_{z, y} \leq N T C_{z, y} \quad \forall z, y
$$

where

$$
\begin{array}{ll}
E_{z, y} & \text { Commercial exchange from zone } \mathrm{z} \text { to zone } \mathrm{y} \\
& {[\mathrm{MW}]}
\end{array}
$$$$
\begin{array}{ll}
N T C_{z, y} & \text { Maximum allowable co } \\
\text { zone } \mathrm{z} \text { to zone y [MW] }
\end{array}
$$

Consider three bidding zones $\mathrm{A}, \mathrm{B}$ and $\mathrm{C}$. Zone $\mathrm{A}$ is connected to both B and C. The ATC trading domain can then be illustrated as in Fig. 1. Each combination of commercial exchanges falling inside the rectangle - defined by the NTC values - is allowed for trading purposes.

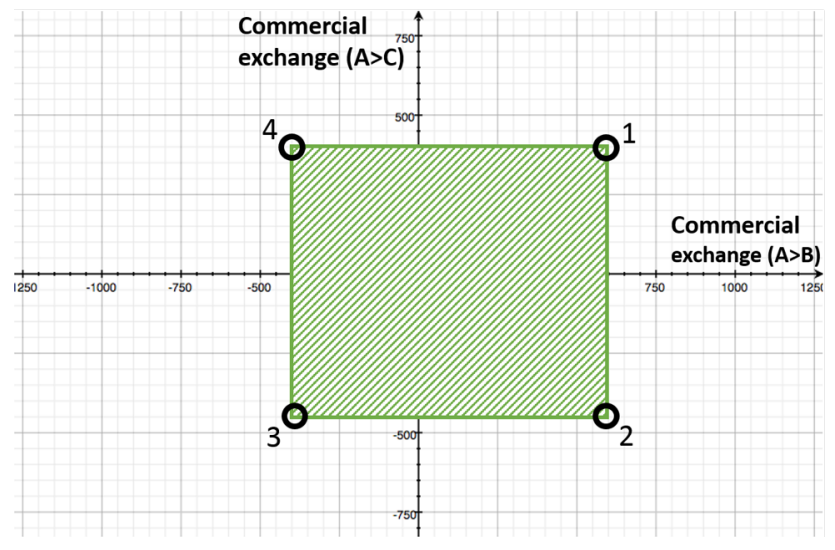

Figure 1: ATC domain

\section{2) Flow-based (FB)}

Instead of supplying fixed commercial capacities, the FB methodology formulates the constraints which reflect the physical limits of the grid. To this end, a simplified network model is constructed, represented by a combination of nodes and lines (Fig. 2). In accordance with the European zonal approach, different nodes are aggregated into zones. Each TSO provides input data for its control area, which is combined at the regional level [9]. The flow-based parameters consist of:

- Critical Branches (CBs): To arrive at a simplified network model without having to consider all individual lines, TSOs each define critical branches (CBs) for their control area. These consist of all crossborder lines, as well as internal lines that are significantly impacted by cross-border exchanges (e.g. highlighted lines in Fig. 2). Only the $\mathrm{CBs}$ are considered in the simplified model. For each $\mathrm{CB}$, the TSO also determines the flow available for the market. This number is based on the thermal limit of the line and furthermore takes into account physical flows that are present on the $\mathrm{CB}$ before market coupling as well as necessary security margins.

- Generation Shift Keys (GSKs): Besides providing data on CBs, TSOs supply a set of Generation Shift Keys (GSKs). A GSK defines how a change in net exchange position of a zone is mapped to altered power output of the generation units within that zone.

- Zonal Power Transfer Distribution Factors (zonal PTDFs): Nodal Power Transfer Distribution Factors linearly describe how nodal injections in each node map to physical flows on the lines of a network. For the purpose of flow-based market coupling, these nodal PTDFs are translated into zonal PTDFs, making use of the defined GSKs. Zonal PTDFs thus denote the physical flow induced on a transmission line, as the result of power injected at a specific zone. Only considering the impact on the defined $\mathrm{CBs}$, it can then be monitored which combinations of cross-zonal exchanges threaten to overload at least one CB.

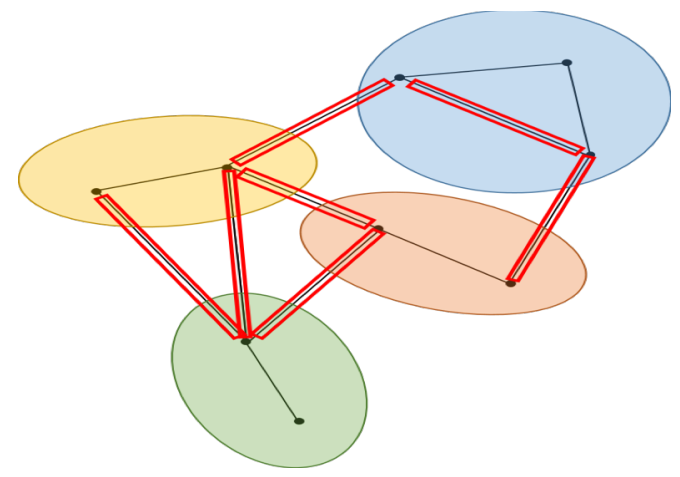

Figure 2: Simplified network model

The combined input data of all individual TSOs leads to a set of restrictions for all CBs. Each constraint limits the combined effect of all zonal net exchange positions to the available flow for each specific CB.

$$
- \text { Fmarket }_{C B} \leq \sum_{z} P T D F_{C B, Z} * N E_{Z} \leq \text { Fmarket }_{C B} \quad \forall C B
$$

where

\footnotetext{
${ }^{3}$ Coreso (COoRdination of Electricity System Operators)
} 


$\begin{array}{ll}P T D F_{C B, z} & \begin{array}{l}\text { Mapping of power injected in zone } \mathrm{z} \text { to } \\ \text { physical flow induced on } \mathrm{CB}[\%]\end{array} \\ N E_{z} & \begin{array}{l}\text { Net commercial exchange position of } \\ \text { zone } \mathrm{z}[\mathrm{MW}]\end{array}\end{array}$

Fmarket $_{C B} \quad$ Flow on CB available for market [MW]

Combining all equations, the derived FB trading domain is illustrated in Fig. 3. This domain corresponds with the global Security of Supply domain, as instead of fixing one NTC capacity value per direction on each border, all constraints imposed by the $\mathrm{CBs}$ are considered. Each constraint is represented by a dotted line in Fig. 3.

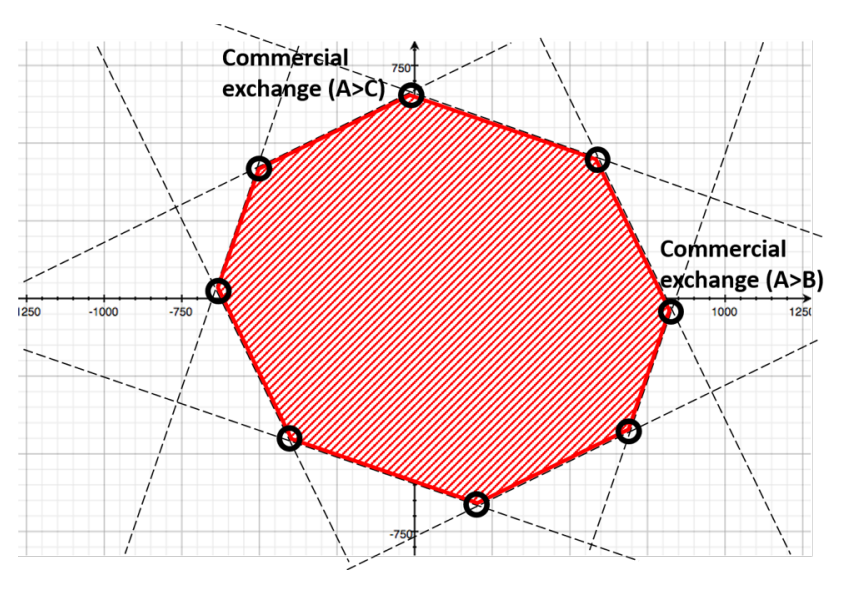

Figure 3: FB domain (Security of Supply domain)

\section{B. Base Case: Verification of Capacity Domain}

The meshed nature of the European power system calls for verification to validate the supplied input data. This verification step is performed two days before delivery and consists of a combination of tests, such as load flow analyses, checking voltage limits of components and assessing voltage stability.

\section{1) Available Transfer Capacity (ATC)}

Under the ATC regime, the daily D2CF file is constructed for only two timestamps, namely for $3 \mathrm{~h} 30$ and $10 \mathrm{~h} 30$. Therefore, two base cases are created. The verification step assesses local grid security and leads to adaptations in case of security breaches. The ATC methodology checks all the NTC corners, which are illustrated in fig. 2. E.g., corner 1 represents a situation in which zone A exports the maximally available commercial capacities or NTC values to both zones B and C.

\section{2) Flow-based (FB)}

In case of flow-based market coupling, the D2CF files are constructed for 24 timestamps as opposed to only 2 timestamps for ATC. Consequently, Coreso composes 24 base cases from the individual D2CF files. The verification step now consists of checking the vertices, instead of the corners under $\mathrm{ATC}^{4}$.

\section{Long-term Adjustments}

At 8h00 day-ahead, more information becomes available as the PTRs market parties plan to use for long-term cross-border transactions have to be nominated/published to the $\mathrm{TSO}^{5}$.

\section{1) Available Transfer Capacity (ATC)}

The Net Transfer Capacity (NTC) is derived from the Total Transfer Capacity (TTC), after deducting a Transfer Reliability Margin (TRM). While the TTC is the maximum commercial exchange possible between two zones in one direction, the TRM is reserved to be able to cope with emergency situations or unexpected deviations in neighbouring zones. Finally, to arrive at the Available Transfer Capacity (ATC) value, the long-term cross-border nominations are subtracted from the NTC value, indicated by the red area in Fig. 4 on the left. The deduced ATC is subsequently available for cross-border trade on the day-ahead market.

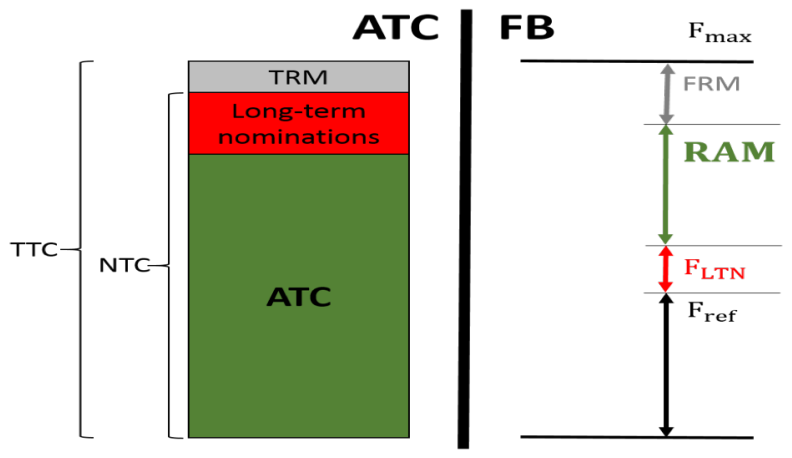

Figure 4: Derivation of ATC (ATC) and RAM (FB)

\section{2) Flow-based (FB)}

A similar approach is implemented to derive the margin available to the market under the FB approach. However, while for ATC an aggregate value per border is taken into account, the FB mechanism considers each line individually. Fig. 4 on the right illustrates the process. Initially, the maximal flow $\left(\mathrm{F}_{\max }\right)$ is available. A security or Flow Reliability Margin (FRM) reflects the uncertainty inherent to the capacity calculation process. $F_{\text {ref }}$ represents the physical flow that will be present on the line due to non-market transactions as forecasted in the base case and a measure for the flows that are not taken into account by the GSK assumption in the zonal market calculation [10]. From the remaining capacity, flows induced by long-term cross-border nominations $\left(\mathrm{F}_{\mathrm{LTN}}\right)$ are subtracted. What can eventually be offered to the day-ahead market is the Remaining Available Margin (RAM) for the considered CB.

\section{Allocation of Available Capacity (Market Coupling)}

For both methodologies, the result of the three preceding pre-coupling steps is a potential trading area. These areas actually denote a combination of constraints, which serve as input to the market coupling algorithm. Taking into account the

\footnotetext{
4 "Vertices" is a broader term used to denote the points that describe the corners or intersections of geometric shapes. Terminologically, it

is convenient to distinguish between "corners" for the ATC domain and "vertices" for the FB domain.

${ }^{5}$ PTR (Physical Transmission Right)
} 
adjustments concerning long-term cross-border nominations, (1) and (2) are transformed as follows:

For ATC:

$$
E_{z, y} \leq A T C_{z, y} \quad \forall z, y
$$

For flow-based:

$$
-R A M_{C B} \leq \sum_{Z} P T D F_{C B, Z} * N E_{Z} \leq R A M_{C B} \quad \forall C B
$$

Comparing both domains in Fig. 5, it is clear that the larger FB domain surrounds the ATC domain. As a consequence, the FB mechanism offers more trading opportunities to the market. Therefore, FB market coupling leads to a solution equal or better in terms of social welfare compared to ATC. Furthermore, when a TSO provides NTC values, he needs to make a choice in advance on how to split the capacity among its borders ( $\mathrm{A}$ to $\mathrm{B}$ and $\mathrm{A}$ to $\mathrm{C}$ ), even before the market participants' bids are known. In contrast, under the FB approach, the entire Security of Supply domain is offered to the market. Driven by bids and offers, the market itself decides on the repartition of commercial capacity among market players.

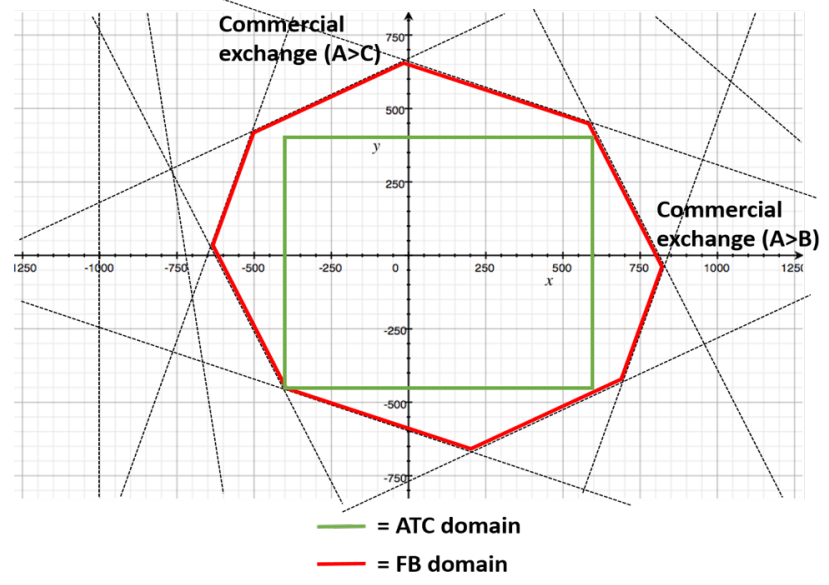

Figure 5: Comparison of ATC versus FB trading domain

\section{Operational Challenges of Flow-BASEd Market COUPLING}

While the flow-based approach is clearly beneficial compared to ATC from a theoretical point of view, incorporating transmission constraints in a zonal pricing approach brings along complications. The main operational challenges inherent to the flow-based mechanism before, during and after market clearing are sequentially highlighted in this section.

\section{A. Pre-coupling challenges}

Zonal flow-based market coupling, just like ATC market coupling, requires the construction of a base case. This is done two days before delivery. For ATC, fixed cross-border exchange limits are calculated during this pre-coupling step. The flow-based mechanism on the other hand determines the

\footnotetext{
${ }^{6}$ Internal trades are exchanges concluded between two market parties which are located inside the same bidding zone.
}

parameters, such as the Generation Shift Keys (GSKs), which are used during market coupling. However, while GSKs forecast the participation of a generation unit to a power shift, this decision actually depends on the market outcome itself, resulting in a circular problem [11]. Furthermore, the formulation of a base case implicitly gives priority to internal trade over cross-border transactions, as the loading of the CBs due to internal trade is assessed already in the base case ${ }^{6}$.

CWE National Regulating Authorities (NRAs) strive for coordination and harmonization regarding the construction of the base case and calculation of the flow-based parameters [11]. Besides, they stress the importance of the base case not to load the grid in a disproportionate way. Finally, efficiently dealing with loop flows, which result from internal trade, further requires an appropriate bidding zone configuration ${ }^{7}[11]$.

\section{B. Market coupling challenges}

A day before delivery, market coupling or day-ahead market clearing is conducted. Under ATC, power is exchanged between two neighbouring countries until price convergence or congestion takes place. On the other hand, under flow-based market coupling, cross-border exchanges between all pairs of countries compete for capacity on each Critical Branch (CB). This intrinsic property of the flow-based mechanism is known as flow factor competition and - unlike ATC - takes into account the impact of a trade on the network, besides the profit of the trade. While being the cornerstone of the flow-based algorithm, in highly stressed situations, adequacy issues may arise. This means buyers who are willing to pay any price to import electricity (but are unable to express this due to price caps in the day-ahead market), are rejected while lower buy bids from other bidding zones are selected. Should this situation emerge, an adequacy patch is in place to prevent the complete isolation of a bidding zone [11], [12].

Moreover, the flow-based algorithm optimizes welfare for an entire region. Therefore, it does not take into account interzonal transactions separately and unintuitive flows, meaning flows from a high to low price region, may occur. Since this raised concern, an adapted algorithm - FB Intuitive market coupling - has been developed, which makes sure no counterintuitive flows take place [13]. Although this correction leads to less overall welfare, it is more acceptable to market parties. Eventually, the decision is made to go live with the intuitive version of the flow-based market coupling algorithm.

\section{Post-coupling challenges}

Since flow-based market coupling is currently only implemented for the day-ahead time frame, intra-day ATC values have to be derived after day-ahead flow-based market coupling. To this end, a coordinated calculation method is implemented, requiring TSOs to coordinate on how to split the capacity among its borders. The regulating authorities of the CWE region formulate in a joint statement their desire for improvements to the intra-day capacity calculation [11]. In expectation of developing a full flow-based capacity calculation and allocation methodology also for the intra-day time frame, they ask for the implementation of intra-day

\footnotetext{
${ }^{7}$ Loop flows are physical flows induced in a bidding zone resulting from internal trade in another bidding zone.
} 
capacity recalculation. This way, more intra-day capacity can be offered when more accurate information concerning the grid, consumption and generation parameters justifies this adaptation.

\section{Example: Price Peaks on Belgian Day-Ahead Market}

Price peaks in combination with a general elevated price level occured on the Belgian day-ahead power exchange Belpex on September $22^{\text {nd }} 2015$ [14]. At the same time, prices in neighbouring zones in the CWE region were significantly lower. The main explanation for this event is found in a precongested base case, caused by a significant amount of internal trade. As such, the remaining capacity for cross-border exchanges on the day-ahead market is severely limited. While this situation can take place also with ATC market coupling in place, flow factor competition may have amplified the price diverging effect. Besides, little to no intra-day capacity on the Belgian borders was left on September $22^{\text {nd }}$, despite the fact that only moderate physical flows were actually observed [14]. This example shows it is imperative to monitor market outcomes under flow-based market coupling, to investigate operational challenges and further improve the flow-based mechanism.

\section{CONCLUSION}

Flow-based market coupling leads to a more efficient use of generation and transmission resources compared to ATC. While under ATC, TSOs themselves determine the commercial capacity available per direction on each border, the flow-based algorithm makes use of the global Security of Supply domain, defined in terms of constraints for all critical branches. Subsequently, taking into account the physical flows induced by each trade, the flow-based algorithm itself decides how transmission capacity is allocated over market parties. More capacity is offered to the market, resulting in a solution equal or better in terms of social welfare.

However, implementing a flow-based mechanism while holding on to zonal pricing is challenging. Pre-coupling, regional coordination and harmonization regarding the base case is essential, as the base case determines the parameters which will be used during flow-based market clearing. Subsequently, the market outcome under flow-based differs from the ATC market outcome. Specific properties of flowbased such as flow factor competition, potentially resulting in adequacy issues, and counter-intuitive flows, have to be taken into account. Finally, flow-based market coupling is currently only implemented for the day-ahead time frame. An extension towards the intra-day time frame is desirable.

In conclusion, while flow-based market coupling offers more trading opportunities on the day-ahead time frame than ATC, it is necessary to follow up and make further improvements regarding the remaining operational challenges. Furthermore, an overall assessment regarding the impact of flow-based market coupling on social welfare, considering both the day-ahead and intra-day time frame, requires further research.

\section{ACKNOWLEDGMENT}

The authors would like to thank Patrick Luickx (CREG) for his valuable input and feedback. However, the final content remains the sole responsibility of the authors.

\section{REFERENCES}

[1] W. W. Hogan, "Contract networks for electric power transmission," J. Regul. Econ., vol. 4, no. 3. pp. 211-242, 1992.

[2] R. Green, "Nodal pricing of electricity: How much does it cost to get it wrong?," J. Regul. Econ., vol. 31, no. 2, pp. 125-149, 2007.

[3] G. Oggioni and Y. Smeers, "Market failures of Market Coupling and counter-trading in Europe: An illustrative model based discussion," Energy Econ., vol. 35, pp. 74-87, 2013.

[4] H. Knops, L. De Vries, and R. Hakvoort, "Congestion management in the European Electricity System: An evaluation of the alternatives," J. Netw. Ind., vol. 1, 2001.

[5] M. Hsu, "An introduction to the pricing of electric power transmission," Util. Policy, vol. 6, no. 3, pp. 257-270, 1997.

[6] Amprion, Apx-Endex, Belpex, Creos, Elia, EnBW, EpexSpot, Rte, and TenneT, (2011,March) CWE Enhanced Flow-Based MC feasibility report. CASC.EU, Luxembourg, Luxembourg. [Online]. Available: $\quad$ http://www.casc.eu/media/CWE\%20FB\%20 Publications/CWE_FB-MC_feasibility_report_2.0_19102011.pdf

[7] Elia, (2009) Modele general de calcul de la capacite de transfert totale et de la marge de fiabilite de transport. Elia, Brussels, Belgium. [Online]. Available: http://www.elia.be/ /media/files/Elia/Products-and-services/Crossborder2/

Year-Month-ModeleGeneralDeCalculDeLaCapacite

DeTransfertTotale.pdf

[8] Elia, (2015, March) Implementation of day-ahead flow-based market coupling in the CWE region General description. Elia, Brussels, Belgium. [Online]. Available: http://www.elia.be/ $\sim /$ media/files/Elia/Projects/Flow-based $\% 20$ market $\% 20$ coupling $\% 2$ 0in\%20Central\%20West\%20Europe/20150313_Description_DA_ FB_MC-NL.pdf

[9] A. Marien, P. Luickx, A. Tirez, and D. Woitrin, "Importance of design parameters on flowbased market coupling implementation," 2013 10th Int. Conf. Eur. Energy Mark., pp. 1-8.

[10] J. Boury, "Methods for the determination of flow-based capacity parameters : description, evaluation and improvements," M.S. thesis, KU Leuven, Leuven, Belgium, 2015.

[11] ACM Bundesnetzagentur, CRE, CREG, E-CONTROL, ILR, (2015, March) Position Paper of CWE NRAs on Flow-Based Market Coupling. CREG, Brussels, Belgium. [Online]. Available: http://www.creg.info/pdf/Opinions/2015/b1410/

CWE_NRA_Position_Paper.pdf

[12] Apx, Belpex, Epex, Elia, Rte, Amprion, TenneT, Creos, and TransnetBW, (2014, November) Adequacy Study CWE. CASC.EU, Luxembourg, Luxembourg. [Online]. Available: http://www.jao.eu/support/resourcecenter/overview?parameters $=\%$ 7B $\% 22$ IsCWEFBMC $\% 22 \% 3 \mathrm{~A} \% 22$ True $\% 22 \% 7 \mathrm{D}$

[13] Apx, Belpex, Epex, Elia, Rte, Amprion, TenneT, Creos, and TransnetBW, (2013, October) CWE Enhanced Flow-Based MC intuitiveness report. [Online]. Available: http://www.elia.be/ $\sim /$ media/files/Elia/Projects/CWE-Flow_Based/ CWE_FB-MC_intuitiveness_report_October_2013.pdf

[14] CREG,$(2015$, November) The price spikes observed on the Belgian day-ahead spot exchange Belpex on 22 September 2015. CREG, Brussels, Belgium. [Online]. Available: http://www.creg.info/pdf/Divers/Z1476EN.pdf 